\title{
Determination of 16 Priority Polycyclic Aromatic Hydrocarbons in Particulate Matter by HRGC-MS after Extraction by Sonication
}

\author{
Pedro Afonso de P. Pereira, ${ }^{* \dagger}$ Jailson B. de Andrade, ${ }^{*}$ and Antonio H. Miguel** \\ *Instituto de Quíimica, Universidade Federal da Bahia-40.170-290, Salvador, BA, Brazil \\ **SCPCS, University of California, Los Angeles-Los Angeles, CA, USA
}

(Received January 30, 2001; Accepted July 19, 2001)

\section{Introduction}

Polycyclic aromatic hydrocarbons (PAH) are semivolatile organic compounds which are emitted, as combustion products, in natural and anthropogenic processes. They are present in the air in the form of vapor or associated with particulate matter, and their role as atmospheric pollutants has been well established by several studies in many groups. ${ }^{1}$ PAH, after chemical or metabolic pathways, are effective carcinogenic/mutagenic agents, thus resulting as the main field of investigation of their biological activities.

The importance of PAH in urban sites is mainly derived from their quantities produced by vehicle emissions, specially heavy duty diesel powered vehicles. According to Harley et al., ${ }^{2}$ the particle phase emission factors, expressed per unit mass of fuel burned, ranged from $21 \mu \mathrm{g} / \mathrm{kg}$ of benzo[ghi]perylene for light duty vehicles to $1000 \mu \mathrm{g} / \mathrm{kg}$ of pyrene for heavy duty diesel vehicles.

Atmospheric particulate matter containing PAH is in general collected over Teflon, glass or quartz fiber filters, followed by an extraction based on Soxhlet, ${ }^{3-5}$ sonication, ${ }^{2,4,6-11}$ microwave, ${ }^{12,13}$ supercritical fluid ${ }^{14}$ or pressurized fluid. ${ }^{5}$

While Soxhlet extraction is time consuming and requires large amounts of toxic and expensive solvents, microwave and supercritical fluid extraction (SFE) require relatively expensive equipment. Sonication, on the other hand, can be done with a minimum amount of extractant solvent and in small time intervals. ${ }^{6}$

In this work, parameters including two types of extractant, extractant volume and time of sonication, are evaluated in the ultrasonic extraction and analysis of $16 \mathrm{PAH}$ from the recently recertificated SRM 1649a, a reference material extensively used for particulate matter. The main objective is to develop a rapid and economical procedure with enough accuracy to estimate, and even quantify, concentrations of PAH in samples of particulate matter. Also, possible PAH losses are evaluated, due to the very usual procedure of concentration by solvent evaporation.

\footnotetext{
$\doteqdot$ To whom correspondence should be addressed

E-mail: pedroapp@ufba.br
}

\section{Experimental}

Material

SRM 1649a Standard Reference Material, Urban Dust (NIST, Colorado, USA): this is an atmospheric particulate material, constituted of dust and collected in an urban area. It is extensively used to evaluate analytical methods for the determination of selected PAH, PCB, chlorinated pesticides, etc.; PAH liquid standard ERS-010 (Cerilliant, Austin, TX): a standard containing the 16 priority components, $10 \mu \mathrm{g} / \mathrm{mL}$ each, in acetonitrile; acetonitrile and dichloromethane, Optima grade (Fisher Scientific, Atlanta); poly(ethylene) syringes and GC microsyringes (Hamilton, NV).

\section{Procedure}

Extractions of SRM 1649a standard were done into $20 \mathrm{~mL}$ screw capped glass flasks, under sonication in a water bath (Fisher Scientific, Atlanta). The conditions for each standard aliquot are given in Table 1. Following the extraction, the resulting suspensions were passed through Millex-FG filter units (PTFE membrane, $0.22 \mu \mathrm{m}$ pore size, $25 \mathrm{~mm} \phi$ ) (Millipore, Bedford) to another glass flask; then $1 \mu \mathrm{L}$ of the filtered solutions were taken up for injection and analysis by GC-MS.

In order to evaluate possible losses due to the evaporation of solutions for PAH concentration, the second sample in Table 1 was additionally treated as described below: a $750 \mu \mathrm{L}$ portion of filtered sample was transferred to a poly(propylene) microcentrifuge tube, evaporated to dryness in a gentle nitrogen stream, redissolved in $750 \mu \mathrm{L}$ of $\mathrm{ACN}$ and then analyzed by GC-MS.

The GC/MS analysis was done in an HP 6890 Plus gas chromatograph, with split/splitless injector, HP 6890 GC autosampler and an HP 5973 quadrupole mass selective detector (Agilent, CA). The column was an HP $1701(30 \mathrm{~m} \times 0.25 \mathrm{~mm})$ (Agilent, CA). The following conditions were employed: i) oven: $60^{\circ} \mathrm{C}(1 \mathrm{~min}) \rightarrow 280^{\circ} \mathrm{C}\left(5^{\circ} \mathrm{C} / \mathrm{min}\right) \rightarrow 280^{\circ} \mathrm{C}(15 \mathrm{~min})$; ii $)$ injector: $270^{\circ} \mathrm{C}$, splitless mode; iii) transfer line: $270^{\circ} \mathrm{C}$; iv) ion source: $230^{\circ} \mathrm{C}$; v) analyzer: $150^{\circ} \mathrm{C}$; vi) electron impact energy: $70 \mathrm{eV}$. Samples were run in SIM mode, in order to enhance the sensitivity.

The quantification was made against an external standard analytical curve, constructed with solutions prepared from the liquid ERS-010 standard in acetonitrile. The $16 \mathrm{PAH}$ determined and the quantitation ions were as follow: 1, naphthalene (NAPH)-128; 2, acenaphthylene (ACNPY)-152; 3, 

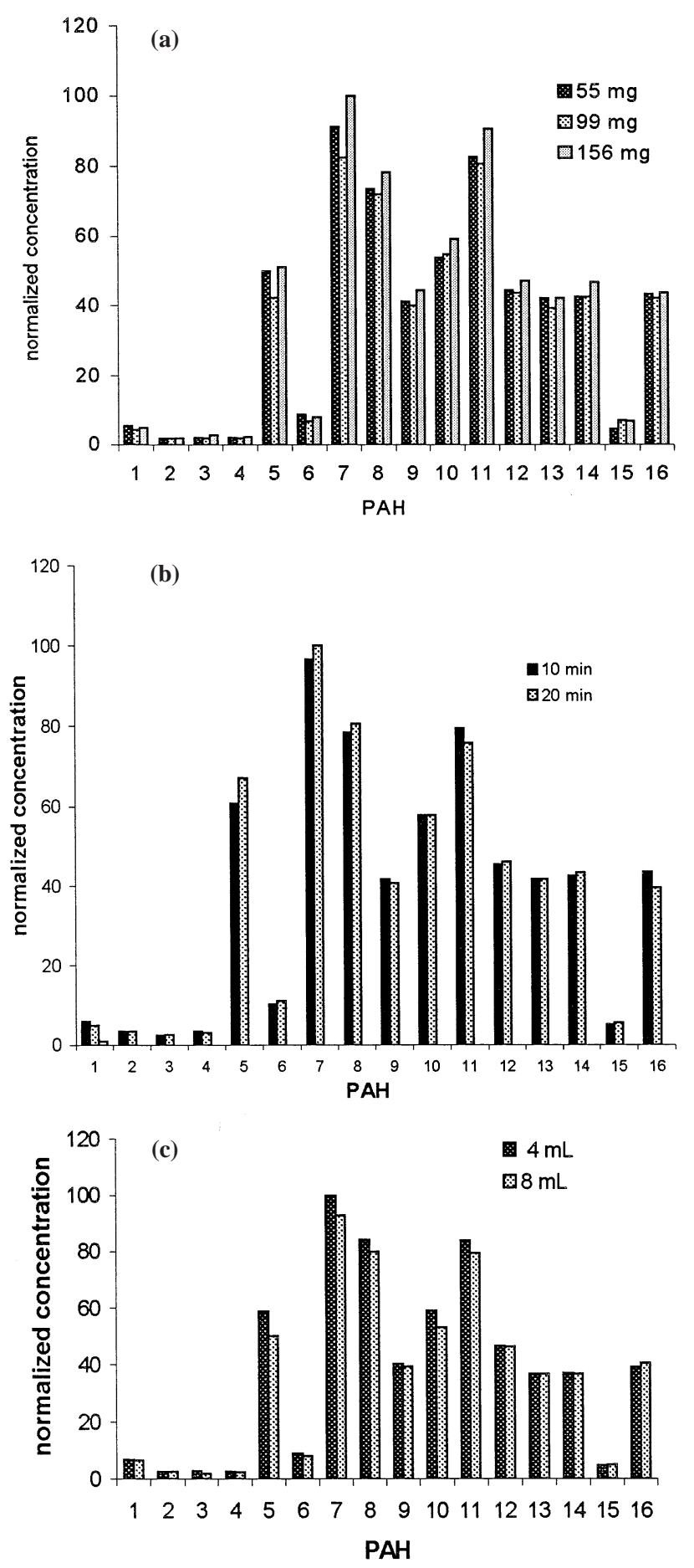

Fig. 1 Influence over extraction of PAH from SRM 1649a by: a) mass of particulate material, b) time, and c) volume of extractant. $\mathrm{PAH}$ concentrations $\left(\mathrm{ng} \mathrm{mg}^{-1}\right.$ ) are plotted normalized to the highest value.

acenaphthene (ACNPE)-154; 4, fluorene (FLUO)-166; 5, phenanthrene (PHE)-178; 6, anthracene (ANTHR)-178; 7, fluoranthene (FLT)-202; 8, pyrene (PYR)-202; 9, benzo[ $a$ ]anthracene (BaA)-228; 10, chrysene (CHRY)-228; 11, benzo[ $b]$ fluoranthene (BbF)-252; 12, benzo[k]fluoranthene (BkF)-252; 13, benzo[a]pyrene (BaP)-252; 14, indeno[1,2,3$c d$ pyrene (IND)-276; 15, dibenz[ah]anthracene (DBA)-278 and 16 , benzo[ $g h i]$ perylene (BGP)-276.
Table 1 Exrtaction conditions for each SRM 1649a aliquot

\begin{tabular}{ccc}
\hline Mass of standard/mg & Extractant $^{\mathrm{a}}$ & $\begin{array}{c}\text { Extraction } \\
\text { time/min }\end{array}$ \\
\hline 55.6 & ACN $(3 \mathrm{~mL})+\mathrm{DCM}(1 \mathrm{~mL})$ & 10 \\
98.6 & ACN $(3 \mathrm{~mL})+\mathrm{DCM}(1 \mathrm{~mL})$ & 10 \\
155.5 & ACN $(3 \mathrm{~mL})+\mathrm{DCM}(1 \mathrm{~mL})$ & 10 \\
49.1 & $\mathrm{DCM}(4 \mathrm{~mL})$ & 10 \\
103.5 & $\mathrm{DCM}(4 \mathrm{~mL})$ & 10 \\
154.5 & $\mathrm{DCM}(4 \mathrm{~mL})$ & 10 \\
165.5 & $\mathrm{DCM}(4 \mathrm{~mL})$ & 20 \\
94.6 & $\mathrm{ACN}(3 \mathrm{~mL})+\mathrm{DCM}(1 \mathrm{~mL})$ & 10 \\
98.2 & ACN $(6 \mathrm{~mL})+\mathrm{DCM}(2 \mathrm{~mL})$ & 10 \\
\hline
\end{tabular}

a. ACN, acetonitrile; DCM, dichloromehane.

\section{Results and Discussion}

Chromatographic analysis of the liquid PAH standard and extracts of the SRM 1649a solid material resulted in the total separation between peaks for all 16 compounds, except BbF and $\mathrm{BkF}$, which elute very close to each other. The total time of analysis is around $53 \mathrm{~min}$. Initially, two types of extractant were evaluated. Namely, three different amounts of the SRM 1649a standard were extracted, each for $10 \mathrm{~min}$, with $4 \mathrm{~mL}$ of DCM. The same procedure was repeated with a mixture of ACN $(3 \mathrm{~mL})$ and DCM $(1 \mathrm{~mL})$. The DCM was added to the $\mathrm{ACN}$ in order to decrease the polarity of the mixture, thus enhancing its extraction power for PAH. The PAH concentrations found, averaged for each set of three extractions, are compared with certified values ${ }^{13}$ in Table 2 . The mixture ACN + DCM appears to be a better extractant than DCM solely for fluoranthene and the next $9 \mathrm{PAH}$, while for the first six, NAPH-ANTHR, results are closely similar for the two extractants employed. Mean recoveries for the 13 certified PAH are, respectively, $79.5 \%$ and $66.1 \%$ for ACN + DCM and DCM as extractants. For fluorene, phenanthrene and benzo[ghi]perylene, the efficiency of the extraction was very low in both extractants. On the other hand, low recovery for benzo[b]fluoranthene and high recovery for benzo[ $k]$ fluoranthene, in ACN + DCM were probably due to peak integration problems.

To evaluate the effect of the total amount of particulate over extraction, three different quantities of the solid standard were sonicated for $10 \mathrm{~min}$, using the mixture of $\mathrm{ACN}(3 \mathrm{~mL})$ and DCM $(1 \mathrm{~mL})$. It can be seen from Fig. 1a (concentrations are shown normalized to $100 \%$, in relation to the highest value determined) that the amount of each PAH extracted did not vary significantly with the mass increase of the standard, suggesting that the volume of extractant used is enough at least for quantities as large as $\sim 160 \mathrm{mg}$ of the standard, representing, according to the certificate, total PAH amounts ranging from 37 ng for fluorene to $1032 \mathrm{ng}$ for fluoranthene and benzo[b]fluoranthene.

Extraction of the standard with sonication, in DCM, over two different time intervals (10 and $20 \mathrm{~min}$ ) was also evaluated. Figure $1 \mathrm{~b}$ shows that increasing extraction time from 10 to 20 min did not increase the amount of PAH extracted from the particulate material. Also, aliquots of the SRM 1649a standard $(\sim 100 \mathrm{mg})$ were extracted for $10 \mathrm{~min}$ with two different volumes of the extractant mixture of ACN and DCM. No increment in $\mathrm{PAH}$ extraction was observed when the volume increased from 4 to $8 \mathrm{~mL}$ (Fig. 1c).

Finally, when a volume of the second sample in Table 1 was 
Table 2 Mean results for 3 extractions in ACN + DCM and DCM, compared to certified concentrations

\begin{tabular}{|c|c|c|c|c|c|}
\hline PAH & $\begin{array}{c}\text { Certified conc. } \\
\text { SRM } 1649 \mathrm{a}^{13} / \\
\mathrm{ng} \mathrm{mg}^{-1}\end{array}$ & $\begin{array}{c}\text { Determined } \\
\text { conc. in the } \\
\text { ACN + DCM } \\
\text { mixture/ } \\
\text { ng mg }^{-1}\end{array}$ & $\% \operatorname{Rec}$ & $\begin{array}{c}\text { Determined } \\
\text { conc. in DCM/ } \\
\text { ng mg } \text { m }^{-1}\end{array}$ & $\% \operatorname{Rec}$ \\
\hline NAPH & - & $0.26 \pm 0.02$ & - & $0.24 \pm 0.06$ & - \\
\hline ACNPY & - & $0.09 \pm 0.00$ & - & $0.14 \pm 0.01$ & - \\
\hline ACNPE & - & $0.11 \pm 0.02$ & - & $0.10 \pm 0.01$ & - \\
\hline FLUO & $0.23 \pm 0.05^{\mathrm{a}}$ & $0.10 \pm 0.01$ & 45.3 & $0.13 \pm 0.03$ & 58.2 \\
\hline PHE & $4.14 \pm 0.37$ & $2.44 \pm 0.2$ & 58.9 & $2.42 \pm 0.26$ & 58.5 \\
\hline ANTHR & $0.43 \pm 0.08$ & $0.40 \pm 0.04$ & 92.4 & $0.41 \pm 0.04$ & 95.4 \\
\hline FLT & $6.45 \pm 0.18$ & $4.65 \pm 0.36$ & 72.1 & $3.85 \pm 0.57$ & 59.6 \\
\hline PYR & $5.29 \pm 0.25$ & $3.80 \pm 0.14$ & 71.8 & $3.13 \pm 0.55$ & 59.0 \\
\hline BAA & $2.21 \pm 0.07$ & $2.13 \pm 0.10$ & 96.6 & $1.67 \pm 0.36$ & 75.8 \\
\hline CHRY & $3.05 \pm 0.06$ & $2.85 \pm 0.12$ & 93.5 & $2.30 \pm 0.30$ & 76.6 \\
\hline $\mathrm{BbF}$ & $6.45 \pm 0.64$ & $4.32 \pm 0.21$ & 66.9 & $3.17 \pm 0.76$ & 49.5 \\
\hline $\mathrm{BkF}$ & $1.91 \pm 0.03$ & $2.30 \pm 0.08$ & 120.5 & $1.81 \pm 0.42$ & 95.3 \\
\hline $\mathrm{BaP}$ & $2.51 \pm 0.09$ & $2.10 \pm 0.07$ & 83.8 & $1.66 \pm 0.39$ & 66.5 \\
\hline IND & $3.18 \pm 0.72$ & $2.24 \pm 0.10$ & 70.3 & $1.70 \pm 0.44$ & 53.0 \\
\hline DBA & $0.29 \pm 0.02$ & $0.31 \pm 0.05$ & 106.6 & $0.21 \pm 0.062$ & 68.5 \\
\hline BGP & $4.01 \pm 0.91$ & $2.20 \pm 0.03$ & 54.9 & $1.74 \pm 0.42$ & 43.4 \\
\hline mean & & & 79.5 & & 66.1 \\
\hline
\end{tabular}

a. Reference concentration.

evaporated and taken up back with $\mathrm{ACN}$, simulating a typical and very common procedure for sample concentration, considerable losses were observed for the first six lightest PAH, ranging from $78 \%$ for naphthalene to $21 \%$ for anthracene. On the other hand, for fluoranthene and the other heaviest $\mathrm{PAH}$, few or no losses were realized. This suggests that, once this concentration procedure is executed on original samples, results for the more volatile PAH could no more be considered as quantitative.

\section{Conclusions}

When compared to other common procedures used in the PAH extraction from particulate material, such as Soxhlet, microwave and supercritical fluid, the use of sonication presents advantages in time and in solvent economy; besides, expensive equipment as in the case of microwave and SFE, is not needed. Also, the use of small quantities of toxic solvents, along with sealed flasks during extraction, minimizes environmental problems due to evaporation.

In this work, parameters such as type of extractant and extraction time and volume were investigated for $16 \mathrm{PAH}$, with two different extractant solvents, using different amounts of a reference particulate material. Results point out that extractions with $4 \mathrm{~mL}$ of a mixture $3: 1$ of ACN and DCM, in $10 \mathrm{~min}$, present a mean recovery of about $80 \%$ of the PAH, in masses of particulate material up to $160 \mathrm{mg}$. On the other hand, an increment in extraction time or volume of extractant did not result in better recoveries.

This procedure is then suitable for simultaneous determination of the 16 priority $\mathrm{PAH}$ present in atmospheric particulate matter, since it presents the advantages of selectivity, quickness, low use of solvents and very little sample preparation needs, with results that can be used to estimate or even quantify their concentration in samples.

Also, when solvent is evaporated in order to concentrate samples, a very common procedure used in quantitation at trace levels, losses for the 6 more volatile PAH are considerable. This should be carefully noted when these compounds are being quantified, pointing to the need of the use of internal references.

\section{Acknowledgements}

The authors would like to thank SEPLANTEC/CADCT and CNPq, Brazil, for financial support and CIAR, USA, for the research fellowship for Pedro Afonso de P. Pereira.

\section{References}

1. W. A. Lopes and J. B. de Andrade, Quim. Nova, 1996, 19(5), 497.

2. L. C. Marr, T. W. Kirchstetter, R. A. Harley, A. H. Miguel, S. V. Hering, and S. K. Hammond, Environ. Sci. Technol., 1999, 33, 3091

3. I. A. Leal-Granadillo, J. I. G. Alonso, and A. Sanz-Medel, J. Environ. Monit., 2000, 2, 218.

4. T. F. Guerin, J. Environ. Monit., 1999, 1, 63.

5. S. A. Wise, L. C. Sander, M. M. Schantz, M. J. Hays, and B. A. Benner, Polyc. Arom. Comp., 2000, 13, 419.

6. A. H. Miguel and J. B. de Andrade, Intern. J. Environ. Anal. Chem., 1989, 35, 35.

7. A. M. Mastral, M. S. Callen, and T. Garcia, Environ. Sci. Technol., 1999, 33, 3177.

8. D. de A. Azevedo, L. S. Moreira, and D. S. de Siqueira, Atmos. Environ., 1999, 33, 4987.

9. J. M. Daisey, A. H. Miguel, J. B. de Andrade, P. A. de P. Pereira, and R. Tanner, J. Air Pol. Control Assoc., 1987 $37,15$.

10. A. H. Miguel, J. B. de Andrade, and S. V. Hering, Intern. J. Environ. Anal. Chem., 1986, 26, 265.

11. A. H. Miguel and P. A. de P. Pereira, Aer. Sci. Technol., 1989, 10, 292.

12. P. Bruno, M. Caselli, G. de Gennaro, M. de Rienzo, and A. Traini, J. Environ. Monit., 2000, 2, 223.

13. H. Budzinski, P. Baumard, A. Papineau, S. Wise, and P. Garrigues, Polyc. Arom. Comp., 1996, 9(1 - 4), 225.

14. O. P. Heemken, B. Stachel, N. Theobald, and B. W. Wenclawiak, Arch. Environ. Contamin. Toxicol., 2000, $38(1), 11$. 